\title{
Intrathecal Administration of Autologous Bone Marrow Mononuclear Cells in a Case of Cerebral Palsy Coexisting with Autistic Features
}

\author{
Sharma $A^{1}$, Sane $\mathbf{H}^{2}$, Pai $\mathrm{S}^{2 *}$, Kulkarni $\mathbf{P}^{2}$, Raichur \\ $\mathbf{M}^{3}$, Kalburgi $\mathbf{S}^{3}$, Inamdar $\mathbf{S}^{3}$, Gokulchandran $\mathbf{N}^{1}$ \\ and Badhe $\mathbf{P}^{4}$ \\ ${ }^{1}$ Department of Medical Services and Clinical research, \\ NeuroGen Brain \& Spine Institute, India \\ ${ }^{2}$ Department of Research \& Development, NeuroGen \\ Brain \& Spine Institute, India \\ ${ }^{3}$ Department of Neurorehabilitation, NeuroGen Brain \& \\ Spine Institute, India \\ ${ }^{4}$ Department of Regenerative Laboratory Services, \\ NeuroGen Brain \& Spine Institute, India \\ *Corresponding author: Suhasini Pai, Department \\ of Research \& Development, NeuroGen Brain \& Spine \\ Institute, Stem Asia Hospital and Research Centre, India
}

Received: November 06, 2016; Accepted: February 17, 2017; Published: February 20, 2017

\begin{abstract}
Cerebral palsy (CP) represents a group of developmental disorders leading to neurodisability in the pediatric population. The 2008 Autism and Developmental Disabilities Monitoring Network (ADDM) CP Network revealed that nearly $7 \%$ of children with CP co-existed with autistic disorders. Here we present a case report of a six and a half year old child who presented with $\mathrm{CP}$ coexisting with autistic features. She underwent two cellular therapies with autologous bone marrow mononuclear administered intrathecally, followed by intensive neurorehabilitation. Over the span of 15 months post cellular therapies, improvements were seen in the walking balance, sitting tolerance, eye contact, clarity of speech, social interaction and voluntary control of the left upper limb with increased weight bearing capacity. There was a decrease from 96 to 82 on the ISAA scale. CARS score reduced from 30.5 to 25 after the two cellular therapies. GMFM score showed an improvement from $64.31 \%$ to $79.96 \%$ and FIM increased from 40 to 49 . No adverse events were observed. This case report supports the evidences suggesting that cellular therapy along with rehabilitation augments the recovery processes in patients with CP coexisting with autistic features. Further clinical studies are required to establish the therapeutic potential of cellular therapy taking into consideration the multifaceted symptomatic presentations in CP.
\end{abstract}

Keywords: Cerebral palsy; Autistic features; Cellular therapy; Autologous bone marrow mononuclear cells

\section{Abbreviations}

CP: Cerebral Palsy; ADDM: Autism and Developmental Disabilities Monitoring Network; ASD: Autism Spectrum Disorders; BMMNC: Bone Marrow Mononuclear Cells; ADL: Activities of Daily Living; FIM: Functional Independence Measure; ISAA: Indian Scale for Assessment of Autism; CARS: Childhood Autism Rating Scale; GMFM: Gross Motor Function Measure; GMFCS: Gross Motor Function Classification System; MRI-DTI: Magnetic Resonance Imaging - Diffusion Tensor Imaging; EEG: Electroencephalogram; ICSCRT: Institutional Committee for Stem Cell Research and Therapy; G-CSF: Granulocyte Colony Stimulating Factor; FACS: Fluorescence Activated Cell Sorting

\section{Case Representation}

A six and a half year old girl was admitted to our hospital with a diagnosis of CP coexisting with autistic features. The patient was born pre-term $\mathrm{C}$-section with breech presentation. At the age of one and half years, she had intermittent convulsions that lasted for 4-5 days following which she entered comatose state and was on ventilator support. The consciousness was regained after 23 days and subsequent mental, social and physical regression was observed in the patient. She had left sided weakness and lost her speech and ability to walk. The patient was started on physiotherapy following which she could walk slowly within a month. Later the patient reached a plateau phase where no further improvements were witnessed in spite of regular physiotherapy.

On assessment prior to cellular therapy, it was observed that the left upper limb was in internal rotation, adduction with flexed elbow and forearm in pronation. Weight bearing capacity was diminished in the left upper extremity with no elicitable tendon reflexes. There was poor voluntary control and scapulothoracic dissociation with grade 2 spasticity in the left upper limb. The attention and concentration was poor with minimum eye contact. Social interaction was poor. There was presence of hyperactivity and the sitting tolerance was average. Emotional responses were inappropriate with presence of behavioral issues like throwing temper tantrums, putting her legs in the mouth and irrelevant laughing. Climbing the stairs was difficult for the patient. Sitting and standing balance was poor. She was dependent for activities of daily living (ADLs). She scored 40 on Functional Independence Measure (Wee-FIM) and 96 on Indian Scale for Assessment of Autism (ISAA) scale. On Childhood Autism Rating Scale (CARS) scale, she was at 30.5. Gross Motor Function Measure (GMFM) score was $76.98 \%$ and the Gross Motor Function Classification System (GMFCS) scale was at level II. Magnetic resonance imaging-Diffusion tensor imaging (MRI- DTI) of the brain showed severe gliotic alterations in almost entire right cerebral hemisphere. The electroencephalogram (EEG) record revealed severely abnormal sleep EEG.
Phys Med Rehabil Int - Volume 4 Issue 1 - 2017 ISSN : 2471-0377 | www.austinpublishing group.com Sharma et al. @ All rights are reserved
Citation: Sharma A, Sane H, Pai S, Kulkarni P, Raichur M, Kalburgi S, et al. Intrathecal Administration of Autologous Bone Marrow Mononuclear Cells in a Case of Cerebral Palsy Coexisting with Autistic Features. Phys Med Rehabil Int. 2017; 4(1): 1110 


\section{Treatment}

The patient underwent autologous bone marrow derived mononuclear cell transplantation. Patient selection was based on World Medical Association Helsinki Declaration for Ethical Principles for medical research involving human subjects [1]. The protocol was reviewed and ethically approved by the Institutional Committee for Stem Cell Research and Therapy (IC-SCRT). A duly filled informed consent form was obtained from the parents prior to the therapy.

G-CSF injections were administrated 72 hours and 24 hours before BMMNCs transplantation. On the day of transplantation, $100 \mathrm{ml}$ of bone marrow was aspirated from the left anterior superior iliac spine. BMMNCs were separated using density gradient separation. The purified MNCs were tested for total cell count, viability and CD34+ cell content by FACS analysis. CD34+ count was found to be 424cells/uL. The cells were administered intrathecally in $\mathrm{L}_{4}-\mathrm{L}_{5}$ space using a lumbar puncture needle. Approximately $1 \times 10^{8}$ BMMNCs with a viability of $98 \%$ were injected. Intravenous administration of Methylprednisolone $1 \mathrm{gm}$ in $500 \mathrm{ml}$ Ringer lactate was carried out simultaneously to improve stem cell survival.

After the transplantation, the patient was put on a multidisciplinary rehabilitation which included physiotherapy, occupational therapy and psychological counseling. These therapies included effective motor learning strategies with task oriented training for successful attainment of functional outcomes. After one week, the patient was discharged.

In view of the improvements observed after the treatment, the patient underwent similar second cellular therapy 10 months after the first intervention. The transplantation procedure was replicated. Total numbers of cells injected were $98 \times 10^{6}$ with $96 \%$ viability.

\section{Results}

The patient was followed up regularly at 1 week, 3 months and 6 months span after first cellular therapy. Within a week of the cellular therapy, improvement in eye contact and sitting tolerance was observed. The hyperactivity had reduced. At 3 months follow up, remarkable improvements were noted in command following, eye contact, attention and concentration. Behavioral issues like throwing temper tantrums, putting her legs in the mouth and irrelevant laughing reduced. Motor coordination, gross and fine motor control had improved which could be noted in various day to day activities. She could perform tandem staircase climbing with minimal support; the balance while sitting and standing had improved. Speech improved to monosyllabic speech. She would try to use her left hand which she could not earlier. Sleep pattern improved. CARS score showed a reduction from 30.5 to 28.5. Wee-FIM improved from 40 to 42 . GMFM score improved from 64.31 to 76.98 . The ISAA score decreased to 92 from 96 .

At 6 months follow up, the voluntary control of the left upper limb improved with increased weight bearing capacity. Pain sensation in the left upper limb was evident and she would cry on getting hurt. She could walk with minimal support. There was further progress in the attention span to 10-15 minutes. Sitting tolerance had further improved and she could attend regular schooling. She would maintain the eye contact during conversation for longer period of time. Clarity of speech had improved significantly. She could recognize and initiate interaction with her relatives and friends. Wee-FIM increased from 42 to 43 and the ISAA score further decreased to 82. CARS score showed further reduction to 27 .

After 10 months of first cellular therapy, she underwent second transplantation based on the improvements observed. At 5 months follow up after second cellular therapy, her static and dynamic balance while sitting and standing further improved. The tightness in the left upper limb reduced and she could perform reach out exercises more easily. She could differentiate between familiar and unfamiliar faces. CARS score showed a further reduction from 27 to 25 . WeeFIM further improved from 43 to 49 . GMFM score further improved from 76.98 to 79.96 .

\section{Discussion}

$\mathrm{CP}$ is one of the most common neurodevelopment conditions affecting the development of movement and posture, which can be attributed to non-progressive disturbances that occurs in the developing fetal or infant brain [2]. While motor impairment is the defining diagnosis basis of $\mathrm{CP}$, the disorder is often associated with cognitive impairment [3]. There are studies suggesting the prevalence of psychological disorders in children with neurodevelopment disorder [4,5]. The 2008 Autism and Developmental Disabilities Monitoring Network (ADDM) CP Network revealed that nearly 7\% of children with CP co-existed with autistic disorders [6]. In children diagnosed with neurodevelopment disorders such as CP in the early stages of their lives, problems with social interaction, communication, and behaviour may be considered as a part of the disorder. So the autistic features may go unnoticed. Early diagnosis and intervention are of critical in the prognosis for children with autistic disorders [7]. The pathophysiology of the brain leading to memory impairment has been linked to structural lesion in basal ganglia, internal capsule and hippocampus [8]. Many of the symptoms associated with CP and coexisting conditions can be managed with a comprehensive treatment plan that addresses the child's primary, secondary and coexisting factors which can help the child live a better life [9]. It has been observed that cellular therapy can be safe and effective in repairing the damage to the neural tissue $[10,11]$.

The patient was administered autologous BMMNC intrathecally. Safety and efficacy of cellular therapy has been proved in previous studies [12]. The autologous cells are preferred over the allogenic cells as the possibility of immune rejection is reduced [13]. BMMNC have a unique property of homing into the damaged areas of the brain and preventing the endogenous cells from further damage [14]. These cells initiate repair processes by inhibiting pro inflammatory cytokines and release of anti- inflammatory molecules and growth factors that are responsible for cell proliferation, cytoprotection, and angiogenesis $[15,16]$.

After intervention, there were marked improvements in voluntary control and sensations in the left upper limb, balance, cognition, social interaction and communication of the patient which were also reflected on the outcome measure scores. Further symptomatic and functional improvements were observed after the second cellular therapy suggesting that multiple doses of BMMNCs 
enhances the therapeutic outcome of therapy. This case also supports the observation that a combination of cellular therapy and exercise results in significant functional improvement through release of neurotrophic factors that stimulate neuronal growth at the site of neurological deficit $[17,18]$. There were no adverse events observed in the patient.

One of the limitations of the case report is the absence of objective imaging studies to determine whether the same brain lesion leading to $\mathrm{CP}$ was also responsible for the autistic pattern seen in the patient Lack of control is another limitation of this case study. But looking at the significant improvement seen after the cellular therapy combined with the multidisciplinary rehabilitation, which was not observed previously with only rehabilitation, the patient could serve as a self control.

\section{Conclusion}

This case study suggests that cellular therapy enhances the outcome of standard rehabilitation treatment by repairing the underlying defect in the brain tissue of $\mathrm{CP}$ coexisting with autistic features. This is possible because cellular therapy addresses both the physical and cognitive deficits. However, larger randomized clinical studies are required to establish the therapeutic effects of cellular therapy taking into consideration the multifaceted symptomatic presentations in CP.

\section{References}

1. World Medical Association. World Medical Association Declaration of Helsinki: Ethical Principles for Medical Research Involving Human Subjects. JAMA. 2013; 310: 2191-2194

2. Rosenbaum $P$, Paneth $N$, Leviton $A$, et al. A report: the definition and classification of cerebral palsy April 2006. Dev Med Child Neurol. 2007; 109 8-14.

3. Nordin V, Gillberg C. Autism spectrum disorders in children with physical or mental disability or both. I: clinical and epidemiological aspects. Dev Med Child Neurol. 1996; 38: 297-313.

4. Himmelmann K, Beckung E, Hagberg G, Uvebrant P. Gross and fine motor function and accompanying impairments in cerebral palsy. Dev Med Child Neurol. 2006; 48: 417-423.430.

5. Sigurdardottir S, Eiriksdottir A, Gunnarsdottir E, Meintema M, Arnadottir U, Vik T. Cognitive profile in young Icelandic children with cerebral palsy. Dev Med Child Neurol. 2008; 50: 357-362.
6. Centers for Disease Control and Prevention. Data and Statistics for Cerebral Palsy.

7. Howlin P. Prognosis in autism: do specialist treatments affect long-term outcome? Eur Child Adolesc Psychiatry. 1997; 6: 55-72.135.

8. Gamayani U, Lestari LP, Machfoed $M H$, Idjradinata $P$, Achmad TH. Relationship between Brain Derived Neurotrophic Factor (BDNF) and cognitive function in children with cerebral palsy. International Journal of Medical and Pharmaceutical Sciences. 2015; 6: 12-16.

9. Jacob VC, Biju H, Sharma A. Neurorehabilitation: A Multidisciplinary Approach. $1^{\text {st }}$ edition. NeuroGen Brain and Spine Institute, Mumbai, India. 2011.

10. Sharma A, Sane H, Gokulchandran N, Kulkarni P, Gandhi S, Sundaram J, et al. A Clinical Study of Autologous Bone Marrow Mononuclear Cells for Cerebral Palsy Patients: A New Frontier. Stem cells international. 2015; 18 : 2015.

11. Sharma A, Sane H, Gokulchandran N, Badhe P, Kulkarni P, Paranjape A. Stem cell therapy for cerebral palsy-a novel option. Cerebral palsy. Challenges for the future. $1^{\text {st }}$ ed. Croatia: In Tech. 2014: 217-242.

12. Sharma A, Gokulchandran N, Chopra G, Kulkarni P, Lohia M, Badhe P, et al. Administration of autologous bone marrow derived mononuclear cells in children with incurable neurological disorders and injury is safe and improves their quality of life. Cell Transplantation. 2012; 21: S1-S12.

13. W Prasongchean and P Ferretti. Autologous stem cells for personalized medicine. Journal of Biotechnology. 2012; 29: 641-650.

14. Sharma A, Sane H, Gokulchandran N, Khopkar D, Paranjape A, Sundaram J, et al. Autologous bone marrow mononuclear cells intrathecal transplantation in chronic stroke. Stroke research and treatment. 2014; 2014

15. Chen PM, Yen ML, Liu KJ, Sytwu HK, Yen BL. Immunomodulatory properties of human adult and fetal multipotent mesenchymal stem cells. Journal of biomedical science. 2011; 18: 49.

16. Li Y, Chen J, Chen XG, Wang L, Gautam SC, Xu YX, et al. Human marrow stromal cell therapy for stroke in rat neurotrophins and functional recovery. Neurology. 2002; 59: 514-523.

17. Carvalho KA, Cunha RC, Vialle EN, Osiecki R, Moreira GH, Simeoni RB, et al Functional Outcome of Bone Marrow Stem Cells (CD45+/CD34-) After Cell Therapy in Acute Spinal Cord Injury: In Exercise Training and in Sedentary Rats. In Transplantation proceedings. 2008; 40: 847-849.

18. Fabel K, Wolf SA, Ehninger D, Babu H, Leal-Galicia P, Kempermann G Additive effects of physical exercise and environmental enrichment on adult hippocampal neurogenesis in mice. Front Neurosci. 2009; 3: 50.
Phys Med Rehabil Int - Volume 4 Issue 1 - 2017

ISSN : 2471-0377 | www.austinpublishinggroup.com

Sharma et al. @ All rights are reserved
Citation: Sharma A, Sane H, Pai S, Kulkarni P, Raichur M, Kalburgi S, et al. Intrathecal Administration of Autologous Bone Marrow Mononuclear Cells in a Case of Cerebral Palsy Coexisting with Autistic Features. Phys Med Rehabil Int. 2017; 4(1): 1110 